\title{
SPECTRAL CONDITIONS FOR JORDAN *-ISOMORPHISMS ON OPERATOR ALGEBRAS
}

\author{
OSAMU HATORI AND LAJOS MOLNÁR
}

\begin{abstract}
In this paper we study non-linear transformations between the unitary groups of von Neumann algebras and the twisted subgroups of positive invertible elements in unital $C^{*}$-algebras with various preserver properties concerning the spectrum, spectral radius, and generalized distance measures. We present several results which show that those transformations are closely related to the Jordan *-isomorphisms between the underlying full algebras. In fact, our results can easily be used for characterizations of that sort of isomorphisms.
\end{abstract}

\section{INTRODUCTION}

In this paper all algebras are assumed to be complex and unital, the unit usually being denoted by 1 . Let $A_{1}, A_{2}$ be algebras and let $\sigma(\cdot)$ stand for the spectrum. A map (no linearity is assumed) $\phi: A_{1} \rightarrow A_{2}$ is called spectrally multiplicative if it satisfies

$$
\sigma(\phi(a) \phi(b))=\sigma(a b)
$$

for all pairs $a, b \in A_{1}$. There has recently been considerable interest in the study of such transformations since in many cases it turns out that they are closely related to isomorphisms, hence the spectral condition displayed above may faithfully compress the linearity and multiplicativity properties of maps into one two-variable equality between sets of scalars. For a typical result we recall that any spectrally multiplicative bijection between the algebras of all continuous complex valued functions over compact Hausdorff spaces is an algebra isomorphism followed by multiplication by a fixed real valued continuous function of modulus 1 . In fact, for first countable spaces this was proved in the paper [7] (which was the starting point of that line of investigations) while in [11] the authors removed the first countability assumption. Concerning operator algebras we obtained in [7] that for an infinite dimensional Hilbert space $H$, any spectrally multiplicative bijection on the algebra of all bounded linear operators on $H$ is either an algebra isomorphism or the negative of an algebra isomorphism. Hence in those cases we have that any spectrally multiplicative bijective map is a transformation which can be written as an algebra isomorphism multiplied by a central symmetry (which is a self-adjoint unitary which is in the center of the algebra in question). (We admit that presently Google Scholar pops up close to 90 citations to the paper [7].) For further reference we mention the survey paper [4] exhibiting a collection of recent results (mainly concerning function algebras) as well as the interesting paper [2] where a variant of spectrally multiplicative maps (involving three variables not only two) has been investigated on general algebras.

In this paper we continue that line of research and present results which can be viewed as characterizations of Jordan *-isomorphisms between operator algebras via their spectral multiplicativity properties of different kinds and their other characteristic invariance properties relating the spectral radius. However, there is significant difference between the previous investigations and the one we report on here. Namely, the properties we consider in this paper are assumed to be satisfied not on the whole algebra but only on certain subsets which are substructures of the general linear group. This means, and again it is the main novelty here, that the spectral multiplicativity and other related conditions are required only on some smaller sets (the so-called twisted subgroup of positive invertible elements, or the unitary group) but, as we shall see, they are still strong

2010 Mathematics Subject Classification. 47B49, 46L99.

Key words and phrases. Jordan *-isomorphism, unitary group, positive invertibles, $C^{*}$-algebra, von Neumann algebra.

The second author was supported by the "Lendület" Program (LP2012-46/2012) of the Hungarian Academy of Sciences and by the Hungarian Scientific Research Fund (OTKA) Reg. No. K115383. 
enough to imply that the transformations under considerations are closely related to Jordan *isomorphisms between the underlying full algebras. In addition to our conditions concerning the spectral radius we investigate transformations which preserve certain distance measures of very general kinds. Furthermore, we study spectral multiplicativity like and other conditions for pairs of maps defined on arbitrary sets with values in the above mentioned substructures of operator algebras.

Before presenting our results we collect the following facts concerning Jordan *-isomorphisms between $C^{*}$-algebras. We first remind that by Proposition 1.3 in [12] every surjective Jordan homomorphism $J$ between arbitrary algebras $A_{1}, A_{2}$ preserves invertibility and satisfies $J\left(a^{-1}\right)=J(a)^{-1}$ for any invertible element $a \in A_{1}$. This implies that a Jordan isomorphism maps the general linear group onto the general linear group and preserves the spectra of elements. We recall the important correspondence between the spectra of the elements $a b$ and $b a$, where $a, b$ belong to an algebra $A$. Namely, we always have $\sigma(a b) \cup\{0\}=\sigma(b a) \cup\{0\}$ and hence, if $a, b$ are invertible, it follows that $\sigma(a b)=\sigma(b a)$. Let now $A_{1}, A_{2}$ be arbitrary $C^{*}$-algebras and $J: A_{1} \rightarrow A_{2}$ be a Jordan isomorphism. By Theorem 6.3.4. in [1] there exists a central projection $q$ (by a projection we always mean a selfadjoint idempotent) in the so-called bounded central closure of $A_{2}$ (a $C^{*}$-algebra that contains $A_{2}$ as a $C^{*}$-subalgebra) such that

$$
J(a b)=q J(a) J(b)+(1-q) J(b) J(a), \quad a, b \in A .
$$

Let $a, b \in A_{1}$ be invertible elements. Denote $x_{1}=q J(a), y_{1}=q J(b)$ and $x_{2}=(1-q) J(a), y_{2}=(1-$ q) $J(b)$. We compute

$$
\sigma(a b) \cup\{0\}=\sigma(J(a b)) \cup\{0\}=\sigma\left(x_{1} y_{1}\right) \cup \sigma\left(y_{2} x_{2}\right)
$$

$$
=\sigma\left(x_{1} y_{1}\right) \cup \sigma\left(x_{2} y_{2}\right)=\sigma(J(a) J(b)) \cup\{0\} .
$$

Since $J$ preserves invertibility, $J(a) J(b)$ is invertible and by the above equality we have

$$
\sigma(a b)=\sigma(J(a) J(b))
$$

which proves that $J$ is spectrally multiplicative on the general linear group.

For a $C^{*}$-algebra $A_{j}$, we denote by $A_{j s}$ the real linear subspace of all self-adjoint elements in $A_{j}$. The set of all positive elements (i.e., self-adjoint elements with non-negative spectrum) in $A_{j}$ is denoted by $A_{j+}$. The set $A_{j+}^{-1}$ of all invertibles in $A_{j+}$ is a so-called twisted subgroup of the general linear group meaning that it is closed under the operation of the inverted Jordan triple product $a b^{-1} a$. For obvious reasons, it is also called the positive definite cone (or positive cone for short). Note that $A_{j+}^{-1}=\exp A_{j s}$. The unitary group of $A_{j}$ is denoted by $U_{j}$. Recall that we have $U_{j}=\exp i A_{j s}$ if $A_{j}$ is a von Neumann algebra. We repeat that by a symmetry we mean a self-adjoint unitary element (or, equivalently a unitary whose square is the identity).

Recall that for the spectral radius $r$ we have the inequality $r(a) \leq\|a\|$ for every $a$ in the $C^{*}$ algebra $A_{j}$ and $r(a)=\|a\|$ holds for any normal element $a \in A_{j}$.

\section{THE CASE OF THE SPACES OF POSITIVE INVERTIBLE ELEMENTS}

Beside characterization via the spectral multiplicativity property, the first main result of the paper, Theorem 4 contains a sort of characterization of Jordan *-isomorphisms in terms of a preserver property relating so-called generalized distance measures. For this we need a recently obtained very general Mazur-Ulam type result that we cite below as Theorem 3. To formulate it we need some preparation. From the paper [8] we recall the following.

Definition 1. Let $X$ be a set equipped with a binary operation $\diamond$ which satisfies the following conditions:

(a1) $a \diamond a=a$ holds for every $a \in X$;

(a2) $a \diamond(a \diamond b)=b$ holds for any $a, b \in X$;

(a3) the equation $x \diamond a=b$ has a unique solution $x \in X$ for any given $a, b \in X$.

In this case the pair $(X, \diamond)$ (or $X$ itself) is called a point-reflection geometry. 
For a $C^{*}$-algebra $A$ and elements $a, b \in A_{+}^{-1}$ define $a \diamond b=a b^{-1} a$. In that way $A_{+}^{-1}$ becomes a point-reflection geometry. Indeed, the conditions (a1), (a2) above are trivial to check. Concerning (a3) we recall that for any given $a, b \in A_{+}^{-1}$, the so-called Ricatti equation $x a^{-1} x=b$ has a unique solution $x=a \# b$ which is just the geometric mean of $a$ and $b$ defined by

$$
a \# b=a^{\frac{1}{2}}\left(a^{-\frac{1}{2}} b a^{-\frac{1}{2}}\right)^{\frac{1}{2}} a^{\frac{1}{2}} .
$$

This result is usually termed as Anderson-Trapp theorem.

We need another concept, the one of so-called generalized distance measures.

Definition 2. Given an arbitrary non-empty set $X$, the function $d: X \times X \rightarrow[0, \infty$ [ is called a generalized distance measure if it has the property that for an arbitrary pair $x, y \in X$ we have $d(x, y)=0$ if and only if $x=y$.

Hence, in this definition we require only the definiteness property of a metric but neither the symmetry nor the triangle inequality is assumed. Our general Mazur-Ulam type theorem in [8] reads as follows.

Theorem 3. Let $X, Y$ be non-empty sets equipped with binary operations $\diamond, \star$, respectively, with which they form point-reflection geometries. Let $d: X \times X \rightarrow[0, \infty[, \rho: Y \times Y \rightarrow[0, \infty[$ be generalized distance measures. Pick $a, b \in X$, set

$$
L_{a, b}=\{x \in X: d(a, x)=d(x, b \diamond a)=d(a, b)\}
$$

and assume the following:

(b1) $d\left(b \diamond x, b \diamond x^{\prime}\right)=d\left(x^{\prime}, x\right)$ holds for all $x, x^{\prime} \in X$;

(b2) $\sup \left\{d(x, b): x \in L_{a, b}\right\}<\infty$;

(b3) there exists a constant $K>1$ such that $d(x, b \diamond x) \geq K d(x, b)$ holds for every $x \in L_{a, b}$.

Let $\phi: X \rightarrow Y$ be a surjective map such that

$$
\rho\left(\phi(x), \phi\left(x^{\prime}\right)\right)=d\left(x, x^{\prime}\right), \quad x, x^{\prime} \in X
$$

and also assume that

(b4) for the element $c \in Y$ with $c \star \phi(a)=\phi(b \diamond a)$ we have $\rho\left(c \star y, c \star y^{\prime}\right)=\rho\left(y^{\prime}, y\right)$ for all $y, y^{\prime} \in Y$. Then we have

$$
\phi(b \diamond a)=\phi(b) \star \phi(a) .
$$

We shall also need the following properties defined for a continuous function $h:] 0, \infty[\rightarrow \mathbb{R}$ :

(c1) $h(t)=0$ holds exactly for $t=1$;

(c2) for some $\theta>0$ real number we have $|h(t)| \geq \theta$ for all $t \in] 0, \infty$ [ from outside a neighborhood of 1 ;

(c3) $h$ is differentiable at $t=1$ and $f^{\prime}(1) \neq 0$;

(c4) $\left|h\left(t_{0}\right)\right| \neq\left|h\left(t_{0}^{-1}\right)\right|$ holds for some $\left.t_{0} \in\right] 0, \infty[$.

The first main result of the paper which involves the possibility of several characterizations of Jordan *-isomorphisms reads as follows.

Theorem 4. Let $A_{j}$ be a $C^{*}$-algebra for $j=1,2$. Suppose that $\phi$ is a surjection from $A_{1+}^{-1}$ onto $A_{2+}^{-1}$. Consider the following statements:

(4.1) $\sigma\left(a b^{-1}\right)=\sigma\left(\phi(a) \phi(b)^{-1}\right), \quad a, b \in A_{1+}^{-1}$;

(4.2) $r\left(a b^{-1}-1\right)=r\left(\phi(a) \phi(b)^{-1}-1\right), \quad a, b \in A_{1+}^{-1}$;

(4.3) there exists a pair $\left.h_{1}, h_{2}:\right] 0, \infty[\rightarrow \mathbb{R}$ of continuous functions which satisfy (c1)-(c3) and we have

$$
\left\|h_{1}\left(b^{-\frac{1}{2}} a b^{-\frac{1}{2}}\right)\right\|=\left\|h_{2}\left(\phi(b)^{-\frac{1}{2}} \phi(a) \phi(b)^{-\frac{1}{2}}\right)\right\|, \quad a, b \in A_{1+}^{-1} ;
$$

(4.4) there exists a Jordan *-isomorphism J from $A_{1}$ onto $A_{2}$, an element $b_{0} \in A_{2+}^{-1}$, a central projection $p \in \mathscr{A}_{2}$ and a positive real number c such that

$$
\phi(a)=b_{0}\left(p J(a)^{c}+(1-p) J(a)^{-c}\right) b_{0}, \quad a \in A_{1+}^{-1} ;
$$


(4.5) there exists a Jordan *-isomorphism J from $A_{1}$ onto $A_{2}$, an element $b_{0} \in A_{2+}^{-1}$, a central projection $p \in \mathscr{A}_{2}$ such that

$$
\phi(a)=b_{0}\left(p J(a)+(1-p) J(a)^{-1}\right) b_{0}, \quad a \in A_{1+}^{-1} ;
$$

(4.6) there exists a Jordan *-isomorphism J from $A_{1}$ onto $A_{2}$ and an element $b_{0} \in A_{2+}^{-1}$ such that

$$
\phi(a)=b_{0} J(a) b_{0}, \quad a \in A_{1+}^{-1} .
$$

We have the implications (4.1) $\Rightarrow(4.2) \Rightarrow(4.3) \Rightarrow(4.4)$. If $h_{1}=h_{2}$, then we have (4.1) $\Rightarrow(4.2) \Rightarrow$ (4.3) $\Rightarrow$ (4.5). If $h_{1}=h_{2}$ and satisfy (c4), then we have (4.1) $\Leftrightarrow(4.2) \Leftrightarrow(4.3) \Leftrightarrow(4.6)$.

Before presenting the proof of Theorem 4 we make some useful remarks. First of all, whenever a normal element of a $C^{*}$-algebra is plugged in a continuous real function (with domain containing the spectrum of that element) that refers to the well-known continuous functional calculus. Let $A$ be a $C^{*}$-algebra.

(R1) If a continuous function $h:] 0, \infty\left[\rightarrow \mathbb{R}\right.$ satisfies (c1), then for any $a \in A_{+}^{-1}$, the equality $h(a)=$ 0 implies that $a=1$. Indeed, by the spectral mapping theorem we have $h(\sigma(a))=\sigma(h(a))=0$, which implies by (c1) that $\sigma(a)=\{1\}$, i.e., $\sigma(a-1)=\{0\}$ which yields $a-1=0$. It follows that the formula

$$
d(a, b)=\left\|h\left(b^{-\frac{1}{2}} a b^{-\frac{1}{2}}\right)\right\|, \quad a, b \in A_{+}^{-1}
$$

defines a generalized distance measure on $A_{+}^{-1}$.

(R2) Observe that if a continuous function $h:] 0, \infty[\rightarrow \mathbb{R}$ satisfies (c1) and (c2), then for any sequence $\left.t_{n} \in\right] 0, \infty$ [ with $h\left(t_{n}\right) \rightarrow 0$ we have $t_{n} \rightarrow 1$. This easily implies that, similarly, for any sequence $a_{n} \in A_{+}^{-1}$ with $h\left(a_{n}\right) \rightarrow 0$ in the norm topology, we have $a_{n} \rightarrow 1$.

(R3) Let $h:] 0, \infty[\rightarrow \mathbb{R}$ be a continuous function which is differentiable at $t=1$. Then the transformation $x \rightarrow h(x), x \in A_{+}^{-1}$ is Fréchet-differentiable at $x=1$ and its derivative $(D h)(1) y=h^{\prime}(1) \cdot y$, $y \in A_{s}$. Indeed, by the differentiability of the real function $h$ we have a continuous function $\omega:] 0, \infty\left[\rightarrow \mathbb{R}\right.$ with $\omega(1)=0$ such that $h(t)-h(1)-h^{\prime}(1)(t-1)=\omega(t)(t-1)$ for all $\left.t \in\right] 0, \infty$ [. It follows that $h(x)-h(1) 1-h^{\prime}(1)(x-1)=\omega(x)(x-1)$ holds for all $x \in A_{+}^{-1}$ from which we obtain that $\left\|h(x)-h(1) 1-h^{\prime}(1)(x-1)\right\| \leq\|\omega(x)\|\|x-1\|$. This implies

$$
\frac{\left\|h(x)-h(1) 1-h^{\prime}(1)(x-1)\right\|}{\|x-1\|} \rightarrow 0
$$

as $x \rightarrow 1$ which proves the assertion.

(R4) If $h:] 0, \infty[\rightarrow \mathbb{R}$ is a continuous function which satisfies (c1) and (c3), then we have a number $K>1$ such that $\left|h\left(t^{2}\right)\right| \geq K|h(t)|$ holds for all $t$ in an $\epsilon$-neighbourhood of 1 with some $0<\epsilon<1$. Indeed, we compute

$$
\frac{h\left(t^{2}\right)}{h(t)}=(t+1) \frac{h\left(t^{2}\right) /\left(t^{2}-1\right)}{h(t) /(t-1)} \rightarrow 2
$$

as $t \rightarrow 1$ verifying our claim.

(R5) For any invertible element $x \in A$ and $a, b \in A_{+}^{-1}$ we have that $a^{\frac{1}{2}} b^{-1} a^{\frac{1}{2}}$ is unitarily equivalent to $b^{-\frac{1}{2}} a b^{-\frac{1}{2}}$ and $\left(x b x^{*}\right)^{-\frac{1}{2}} x a x^{*}\left(x b x^{*}\right)^{-\frac{1}{2}}$ is unitarily equivalent to $b^{-\frac{1}{2}} a b^{-\frac{1}{2}}$. Indeed, as for the former statement, we have

$$
a^{\frac{1}{2}} b^{-1} a^{\frac{1}{2}}=u^{*}\left(b^{-\frac{1}{2}} a b^{-\frac{1}{2}}\right) u,
$$

where $u$ is the unitary element in the polar decomposition of $b^{-\frac{1}{2}} a^{\frac{1}{2}} \in A$. As for the latter statement, we have

$$
\left(x b x^{*}\right)^{-\frac{1}{2}} x a x^{*}\left(x b x^{*}\right)^{-\frac{1}{2}}=\left|b^{\frac{1}{2}} x^{*}\right|^{-1}\left(b^{\frac{1}{2}} x^{*}\right)^{*}\left(b^{-\frac{1}{2}} a b^{-\frac{1}{2}}\right)\left(b^{\frac{1}{2}} x^{*}\right)\left|b^{\frac{1}{2}} x^{*}\right|^{-1}
$$

$$
=v^{*}\left(b^{-\frac{1}{2}} a b^{-\frac{1}{2}}\right) \nu
$$

where $v$ is the unitary element in the polar decomposition of $b^{\frac{1}{2}} x^{*}$. 
(R6) For any scalar valued continuous function $h$ on $] 0, \infty$ [, unitary $u \in A$ and positive invertible $a \in A_{+}^{-1}$ we have $h\left(u a u^{-1}\right)=u h(a) u^{-1}$. Indeed, it follows easily from the fact that $h$ can be uniformly approximated by polynomials on any compact subinterval of $] 0, \infty$ [ and from the isometric property of the continuous functional calculus.

Proof of Theorem 4. It is obvious that (4.1) implies (4.2). To verify the implication (4.2) $\Rightarrow$ (4.3) observe that for any $a, b \in A_{1+}^{-1}$ we have

$$
\sigma\left(a b^{-1}-1\right)=\sigma\left(a b^{-1}\right)-1=\sigma\left(b^{-\frac{1}{2}} a b^{-\frac{1}{2}}\right)-1=\sigma\left(b^{-\frac{1}{2}} a b^{-\frac{1}{2}}-1\right)
$$

which implies that

$$
r\left(a b^{-1}-1\right)=r\left(b^{-\frac{1}{2}} a b^{-\frac{1}{2}}-1\right)=\left\|b^{-\frac{1}{2}} a b^{-\frac{1}{2}}-1\right\| .
$$

Therefore, assuming (4.2) and defining $\left.h_{1}(t)=h_{2}(t)=t-1, t \in\right] 0, \infty$ [ we plainly obtain (4.3).

The main part of the proof now follows. We assume that (4.3) holds with continuous functions $\left.h_{1}, h_{2}:\right] 0, \infty[\rightarrow \mathbb{R}$ satisfying (c1)-(c3). Define

$$
d(a, b)=\left\|h_{1}\left(b^{-\frac{1}{2}} a b^{-\frac{1}{2}}\right)\right\|, \quad a, b \in A_{1+}^{-1}
$$

and

$$
\rho(a, b)=\left\|h_{2}\left(b^{-\frac{1}{2}} a b^{-\frac{1}{2}}\right)\right\|, \quad a, b \in A_{2+}^{-1} .
$$

By the remark (R1) we know that $d, \rho$ are generalized distance measures and we have

$$
\rho(\phi(a), \phi(b))=d(a, b), \quad a, b \in A_{1+}^{-1} .
$$

Observe the following. Applying (R5) and (R6) we have

$$
d\left(z a z^{*}, z b z^{*}\right)=d(a, b)
$$

and

$$
d\left(b x^{-1} b, b x^{\prime-1} b\right)=d\left(x^{-1}, x^{\prime-1}\right)=d\left(x^{\prime}, x\right)
$$

for all $a, b, x \in A_{1+}^{-1}$ and invertible $z \in A_{1}$. Clearly, similar properties hold for the generalized distance measure $\rho$, too.

Now define the map $\phi_{0}: A_{1+}^{-1} \rightarrow A_{2+}^{-1}$ by $\phi_{0}(a)=\phi(1)^{-\frac{1}{2}} \phi(a) \phi(1)^{-\frac{1}{2}}, a \in A_{1+}^{-1}$. Plainly, $\phi_{0}$ is a well defined and surjective map from $A_{1+}^{-1}$ onto $A_{2+}^{-1}, \phi_{0}$ is unital meaning that $\phi_{0}(1)=1$, and the equality (1) holds also for $\phi_{0}$, i.e., we have $\rho\left(\phi_{0}(a), \phi_{0}(b)\right)=d(a, b), a, b \in A_{1+}^{-1}$.

We are going to apply Theorem 3 . In order to do that we need to check that the conditions in that theorem are satisfied. Firstly, we define the point-reflection geometry structures on $A_{j+}^{-1}$ in the standard way, i.e., just as we did after Definition 1. The condition (b1) is fulfilled by (2).

To proceed, we claim the following. Let $H$ be a subset of $A_{1+}^{-1}$ with the property that there are positive numbers $\alpha, \beta$ such that $\alpha 1 \leq y \leq \beta 1$ holds for all $y \in H$. (This means that $H$ is bounded away from zero and also from above with respect to the usual order $\leq$ defined on the set of all selfadjoint elements coming from the notion of positivity. Recall that positive elements are the selfadjoint ones with spectrum within the set of non-negative reals.) Then we assert that there exists a number $\delta>0$ with the property that whenever $a, b \in H$ are such that $\|a-b\|<\delta$, we necessarily have $\left\|b^{-\frac{1}{2}} x b^{-\frac{1}{2}}-1\right\|<\epsilon$ (i.e., the spectrum of $b^{-\frac{1}{2}} x b^{-\frac{1}{2}}$ is in $] 1-\epsilon, 1+\epsilon$ [) for all $x \in L_{a, b}$, where $\epsilon$ is the number that appears in (R4) in relation with $h_{1}$. Assume for a moment that this assertion is already proven. We can check rather easily that for $a, b \in A_{1+}^{-1}$ with $\|a-b\|<\delta$ the properties (b2) and (b3) are satisfied. Indeed, by the isometric property of the continuous functional calculus, the fulfillment of (b2) is clear since $h_{1}$ is bounded in the closed interval $[1-\epsilon, 1+\epsilon]$. As for (b3), applying the second equality in (2), the first part of (R5) and (R6), we can compute

$$
\begin{aligned}
d\left(x, b x^{-1} b\right)=d\left(b^{-1} x b^{-1}, x^{-1}\right)=\left\|h_{1}\left(x^{\frac{1}{2}}\left(b^{-1} x b^{-1}\right) x^{\frac{1}{2}}\right)\right\| \\
=\left\|h_{1}\left(\left(x^{\frac{1}{2}} b^{-1} x^{\frac{1}{2}}\right)^{2}\right)\right\|=\left\|h_{1}\left(\left(b^{-\frac{1}{2}} x b^{-\frac{1}{2}}\right)^{2}\right)\right\| \geq K\left\|h_{1}\left(b^{-\frac{1}{2}} x b^{-\frac{1}{2}}\right)\right\|=K d(x, b)
\end{aligned}
$$

for all $x \in L_{a, b}$ meaning that (b3) is also satisfied. 
Now, in order to verify the starting assertion, assume on the contrary that we have sequences $a_{n}, b_{n} \in H, x_{n} \in L_{a_{n}, b_{n}}$ such that $\left\|a_{n}-b_{n}\right\|<\frac{1}{n}$ but $\left\|b_{n}^{-\frac{1}{2}} x_{n} b_{n}^{-\frac{1}{2}}-1\right\| \geq \epsilon$. We compute

$$
\left\|b_{n}^{-\frac{1}{2}} a_{n} b_{n}^{-\frac{1}{2}}-1\right\|=\left\|b_{n}^{-\frac{1}{2}}\left(a_{n}-b_{n}\right) b_{n}^{-\frac{1}{2}}\right\| \leq\left\|b_{n}^{-1}\right\|\left\|a_{n}-b_{n}\right\|
$$

and this last term converges to 0 since we have $\left\|b_{n}^{-1}\right\| \leq 1 / \alpha$. Therefore, $b_{n}^{-\frac{1}{2}} a_{n} b_{n}^{-\frac{1}{2}} \rightarrow 1$ and by (c1) it follows that $d\left(a_{n}, b_{n}\right)=\left\|h_{1}\left(b_{n}^{-\frac{1}{2}} a_{n} b_{n}^{-\frac{1}{2}}\right)\right\| \rightarrow 0$. Since $x_{n} \in L_{a_{n}, b_{n}}$, we have $d\left(a_{n}, x_{n}\right)=d\left(a_{n}, b_{n}\right) \rightarrow 0$ meaning that $h_{1}\left(x_{n}^{-\frac{1}{2}} a_{n} x_{n}^{-\frac{1}{2}}\right) \rightarrow 0$. Applying the observation in (R2) one can check rather easily that this implies $x_{n}^{-\frac{1}{2}} a_{n} x_{n}^{-\frac{1}{2}} \rightarrow 1$. Therefore, for an arbitrary scalar $0<\gamma<1$ we have an index $n_{0}$ such that for all $n \geq n_{0}$ we obtain $1-\gamma 1 \leq x_{n}^{-\frac{1}{2}} a_{n} x_{n}^{-\frac{1}{2}} \leq 1+\gamma 1$ which yields $(1 /(1+\gamma)) a_{n} \leq x_{n} \leq$ $(1 /(1-\gamma)) a_{n}$ for all $n \geq n_{0}$. Since we also have $b_{n}^{-\frac{1}{2}} a_{n} b_{n}^{-\frac{1}{2}} \rightarrow 1$, in a similar manner, we may also assume that $(1 /(1+\gamma)) a_{n} \leq b_{n} \leq(1 /(1-\gamma)) a_{n}$ holds for all $n \geq n_{0}$. These imply that

$$
(1 /(1+\gamma)-1 /(1-\gamma)) a_{n} \leq x_{n}-b_{n} \leq(1 /(1-\gamma)-1 /(1+\gamma)) a_{n}
$$

for all $n \geq n_{0}$. Since $\gamma>0$ is arbitrary and we have $a_{n} \leq \beta 1$ for all $n$, we infer that $x_{n}-b_{n} \rightarrow 0$ which immediately yields $b_{n}^{-\frac{1}{2}} x_{n} b_{n}^{-\frac{1}{2}} \rightarrow 1$ contradicting to $\left\|b_{n}^{-\frac{1}{2}} x_{n} b_{n}^{-\frac{1}{2}}-1\right\| \geq \epsilon$. This proves the above assertion and hence we have that (b2) and (b3) in Theorem 3 are satisfied. Observe that (b4) is fulfilled, too, which can be checked just as the condition (b1) above. By Theorem 3 it follows that there is a number $\delta>0$ such that whenever $\|a-b\|<\delta, a, b \in A_{1+}^{-1}$, we necessarily have

$$
\phi_{0}\left(b a^{-1} b\right)=\phi_{0}(b) \phi_{0}(a)^{-1} \phi_{0}(b) .
$$

Now pick arbitrary $a, b \in A_{1+}^{-1}$. We prove that the above displayed equality holds for $a$ and $b$. To verify this, consider the curve

$$
\Gamma(t)=a^{\frac{1}{2}}\left(\exp \left(t \log \left(a^{-\frac{1}{2}} b a^{-\frac{1}{2}}\right)\right)\right) a^{\frac{1}{2}}, \quad t \in[0,2]
$$

connecting $a$ and $b a^{-1} b$ and passing through $b$. The range of this curve is a norm-compact subset of $A_{1+}^{-1}$ and hence it satisfies the condition we imposed on the subset $H$ of $A_{1+}^{-1}$ in the previous part of the proof. Therefore, there is a number $\delta>0$ such that for any $a^{\prime}, b^{\prime} \in \Gamma([0,2])$ we have $\phi_{0}\left(b^{\prime} a^{\prime-1} b^{\prime}\right)=\phi_{0}\left(b^{\prime}\right) \phi_{0}\left(a^{\prime}\right)^{-1} \phi_{0}\left(b^{\prime}\right)$. By the uniform continuity of $\Gamma$, for close enough $t, s \in[0,2]$ we have $\|\Gamma(t)-\Gamma(s)\|<\delta$. Now, we can select a large enough $n$ such that for the elements $a_{k}=\Gamma\left(k / 2^{n}\right)$, $k=0,1, \ldots, 2^{n+1}$ we have $\left\|a_{k}-a_{k+1}\right\|<\delta$. Clearly, $a_{0}=a, a_{2^{n}}=b, a_{2^{n+1}}=b a^{-1} b$, and $a_{k+1} a_{k}^{-1} a_{k+1}=$ $a_{k+2}$ holds for every $0 \leq k \leq 2^{n+1}-2$. Moreover, by the closeness of $a_{k}$ and $a_{k+1}$ we have

$$
\phi_{0}\left(a_{k+1} a_{k}^{-1} a_{k+1}\right)=\phi_{0}\left(a_{k+1}\right) \phi_{0}\left(a_{k}\right)^{-1} \phi_{0}\left(a_{k+1}\right)
$$

for every $0 \leq k \leq 2^{n+1}-1$. It requires purely algebraic computations to verify that then we have

$$
\phi_{0}\left(a_{2^{n}} a_{0}^{-1} a_{2^{n}}\right)=\phi_{0}\left(a_{2^{n}}\right) \phi_{0}\left(a_{0}\right)^{-1} \phi_{0}\left(a_{2^{n}}\right) .
$$

In fact, this is just the content of the technical Lemma 4.2 in [3]. As $a_{0}=a$ and $a_{2^{n}}=b$, we get

$$
\phi_{0}\left(b a^{-1} b\right)=\phi_{0}(b) \phi_{0}(a)^{-1} \phi_{0}(b)
$$

for an arbitrary pair $a, b \in A_{1+}^{-1}$.

Putting $b=1$ we deduce that $\phi_{0}\left(a^{-1}\right)=\phi_{0}(a)^{-1}$ for every $a \in A_{1+}^{-1}$ and then we obtain that

$$
\phi_{0}(b a b)=\phi_{0}(b) \phi_{0}(a) \phi_{0}(b), \quad a, b \in A_{1+}^{-1} .
$$

One can trivially deduce using (3) and (4) that for any $a \in A_{1+}^{-1}$ we have $\phi_{0}\left(a^{m}\right)=\phi_{0}(a)^{m}$ first for any integer $m$ and then for any rational number, too.

Pick $x \in A_{1 s}$ and define $S: \mathbb{R} \rightarrow A_{2+}^{-1}$ by

$$
S(t)=\phi_{0}(\exp (t x)), \quad t \in \mathbb{R} .
$$


We assert that $S$ is continuous with respect the norm-topology. To see this, first observe that applying (R2) for a sequence $x_{n} \in A_{1+}^{-1}$ we have

$$
\begin{aligned}
& \left\|x_{n}-1\right\| \rightarrow 0 \Rightarrow\left\|h_{1}\left(x_{n}\right)\right\| \rightarrow 0 \Rightarrow d\left(x_{n}, 1\right) \rightarrow 0 \Rightarrow \\
& \quad \rho\left(\phi_{0}\left(x_{n}\right), \phi_{0}(1)\right)=\rho\left(\phi_{0}\left(x_{n}\right), 1\right) \rightarrow 0 \Rightarrow\left\|h_{2}\left(\phi_{0}\left(x_{n}\right)\right)\right\| \rightarrow 0 \Rightarrow\left\|\phi_{0}\left(x_{n}\right)-1\right\| \rightarrow 0 .
\end{aligned}
$$

Now, picking $t, t_{0} \in \mathbb{R}$ we compute

$$
\begin{aligned}
& \left\|S\left(t+t_{0}\right)-S\left(t_{0}\right)\right\|=\left\|\phi_{0}\left(\exp \left(\left(t+t_{0}\right) x\right)\right)-\phi_{0}\left(\exp \left(t_{0} x\right)\right)\right\| \\
& \leq\left\|\phi_{0}\left(\exp \left(t_{0} x\right)\right)^{\frac{1}{2}}\right\|^{2}\left\|\phi_{0}\left(\exp \left(t_{0} x\right)\right)^{-\frac{1}{2}} \phi_{0}\left(\exp \left(\left(t+t_{0}\right) x\right)\right) \phi_{0}\left(\exp \left(t_{0} x\right)\right)^{-\frac{1}{2}}-1\right\| \\
& =\left\|\phi_{0}\left(\exp \left(t_{0} x\right)\right)\right\|\left\|\phi_{0}\left(\exp \left(\frac{-t_{0}}{2} x\right) \exp \left(\left(t+t_{0}\right) x\right) \exp \left(\frac{-t_{0}}{2} x\right)\right)-1\right\| \\
& =\left\|\phi_{0}\left(\exp \left(t_{0} x\right)\right)\right\|\left\|\phi_{0}(\exp (t x))-1\right\| .
\end{aligned}
$$

It follows that for $t \rightarrow 0$ we have $S\left(t+t_{0}\right) \rightarrow S\left(t_{0}\right)$ in the norm topology implying the norm-continuity of $S$. We next deduce that $S$ is a one-parameter group in $A_{2+}^{-1}$. Indeed, let $m, n, m^{\prime}, n^{\prime}$ be integers with $m, m^{\prime} \neq 0$. We calculate

$$
\begin{aligned}
S\left(\frac{n}{m}+\frac{n^{\prime}}{m^{\prime}}\right)=\phi_{0}\left(\exp \left(\left(\frac{n}{m}+\frac{n^{\prime}}{m^{\prime}}\right) x\right)\right) & =\phi_{0}\left(\exp \frac{1}{m m^{\prime}} x\right)^{m^{\prime} n+m n^{\prime}} \\
& =\phi_{0}\left(\exp \frac{1}{m m^{\prime}} x\right)^{m^{\prime} n} \phi_{0}\left(\exp \frac{1}{m m^{\prime}} x\right)^{m n^{\prime}}=S\left(\frac{n}{m}\right) S\left(\frac{n^{\prime}}{m^{\prime}}\right) .
\end{aligned}
$$

Since $S$ is continuous, it follows that

$$
S\left(t+t^{\prime}\right)=S(t) S\left(t^{\prime}\right), \quad t, t^{\prime} \in \mathbb{R} .
$$

Therefore, $S$ is a continuous one-parameter group in $A_{2}$. By part (a) in Proposition 6.4.6 in [10] there exists $y \in A_{2}$ with

$$
S(t)=\exp (t y), \quad t \in \mathbb{R} .
$$

Since $S(t)$ is self-adjoint, we deduce that $y$ is also self-adjoint using, e.g., (c) in Proposition 6.4.6 in [10].

Defining $f(x)=y$ we obtain a map $f: A_{1 s} \rightarrow A_{2 s}$ for which

$$
\phi_{0}(\exp (t x))=S(t)=\exp (t f(x)), \quad t \in \mathbb{R}, x \in A_{1 s} .
$$

As $\phi_{0}$ preserves or, better say, respects the pair $d, \rho$ of generalized distance measures, it is clearly injective. This implies that $f$ is injective, too. Considering $\phi_{0}^{-1}$ in the place of $\phi_{0}$, we clearly have an injective map $g: A_{2 s} \rightarrow A_{1 s}$ such that $\phi_{0}^{-1}(\exp (t y))=\exp (\operatorname{tg}(y))$ holds for every $y \in A_{2 s}$ and $t \in \mathbb{R}$. This easily implies that $y=f(g(y))$ holds for all $y \in A_{2 s}$. Hence $f$ is surjective and therefore it is a bijection from $A_{1 s}$ onto $A_{2 s}$. Note that $f(0)=0$ is true by the definition of $f$.

Our next claim is that $f$ is a scalar multiple of a norm-isometry. To verify this, we assert that as $t \rightarrow 0$, we have that

$$
\frac{d(\exp (t x), \exp (t y))}{|t|} \rightarrow\left|h_{1}^{\prime}(1)\right|\|x-y\|
$$

holds for all $x, y \in A_{1 s}$. Clearly,

$$
\frac{\exp \left(-\frac{t}{2} y\right) \exp (t x) \exp \left(-\frac{t}{2} y\right)-1}{t}
$$

$$
=\exp \left(-\frac{t}{2} y\right) \frac{(\exp (t x)-1)-(\exp (t y)-1)}{t} \exp \left(-\frac{t}{2} y\right) \rightarrow x-y \text {. }
$$

Since

$$
\frac{d(\exp (t x), \exp (t y))}{|t|}=\frac{\left\|h_{1}\left(\exp \left(-\frac{t}{2} y\right) \exp (t x) \exp \left(-\frac{t}{2} y\right)\right)-h_{1}(1)\right\|}{|t|}
$$


the validity of (5) follows from (R3) and from the chain rule. Similarly, we obtain that

$$
\frac{\rho(\exp (t x), \exp (t y))}{|t|} \rightarrow\left|h_{2}^{\prime}(1)\right|\|x-y\|
$$

holds for all $x, y \in A_{2 s}$. Since $\phi_{0}$ respects the pair $d, \rho$ of generalized distance measures, it now follows that $\left|h_{1}^{\prime}(1)\right|\|x-y\|=\left|h_{2}^{\prime}(1)\right|\|f(x)-f(y)\|, x, y \in A_{1 s}$. This implies that we have a positive scalar $c$ such that $(1 / c) f$ is a surjective isometry from $A_{1 s}$ onto $A_{2 s}$. Since $f(0)=0$, by the classical Mazur-Ulam theorem we infer that $f$ is linear. The structure of linear isometries between the selfadjoint parts of $C^{*}$-algebras is well-known. In fact, according to a theorem of Kadison [6, Therem 2] we obtain that $(1 / c) f(1)$ is a central symmetry in $A_{2}$ and there is a Jordan *-isomorphism $J$ from $A_{1}$ onto $A_{2}$ such that

$$
f(x)=f(1) J(x), \quad x \in A_{1 s} .
$$

Put $p=(1+(1 / c) f(1)) / 2$. Then $p$ is a central projection in $A_{2}$ and

$$
f(x)=c(p J(x)-(1-p) J(x)), \quad x \in A_{1 s} .
$$

We now calculate

$$
\begin{aligned}
& \phi_{0}(\exp x)=\exp (c(p J(x)-(1-p) J(x))) \\
& =\sum_{n=0}^{\infty} \frac{(c(p J(x)-(1-p) J(x)))^{n}}{n !}=\sum_{n=0}^{\infty} \frac{p J\left((c x)^{n}\right)+(1-p) J\left((-c x)^{n}\right)}{n !} \\
& \quad=p J(\exp (c x))+(1-p) J(\exp (-c x))=p J(\exp x)^{c}+(1-p) J(\exp x)^{-c}
\end{aligned}
$$

for every $x \in A_{1 s}$. Thus

$$
\phi_{0}(a)=p J(a)^{c}+(1-p) J(a)^{-c}, \quad a \in A_{1+}^{-1},
$$

and we arrive at (4.4). Observe further that if we assume $h_{1}=h_{2}$ and that the central projection $p$ above is non-trivial, then inserting $a=t 1, t \in] 0, \infty$ [ and $b=1$ into (6), and using the generalized distance measure preserving property of $\phi_{0}$, we easily obtain

$$
\left|h_{1}(t)\right|=\max \left\{\left|h_{1}\left(t^{c}\right)\right|,\left|h_{1}\left(t^{-c}\right)\right|\right\}
$$

for all positive real number $t$. From this we first deduce that $\left|h_{1}(t)\right|=\left|h_{1}\left(t^{-1}\right)\right|$ and then that $\left.\left|h_{1}(t)\right|=\left|h_{1}\left(t^{c}\right)\right|, t \in\right] 0, \infty$ [. Differentiating $h_{1}$ at $t=1$ we easily obtain that $c=1$. Therefore, in the case where the projection $p$ is non-trivial, we have $c=1$. Similar argument applies when $p$ is trivial, i.e., when $\left|h_{1}(t)\right|=\left|h_{1}\left(t^{c}\right)\right|$ or $\left.\left|h_{1}(t)\right|=\left|h_{1}\left(t^{-c}\right)\right|, t \in\right] 0, \infty$ [. This gives us the implication $(4.3) \Rightarrow(4.5)$.

If above we also assume that $h_{1}=h_{2}$ and $\left|h_{1}\left(t_{0}\right)\right| \neq\left|h_{1}\left(t_{0}^{-1}\right)\right|$ holds for some $\left.t_{0} \in\right] 0, \infty$, then reconsidering the last part of the argument above, we see that $p$ is necessarily trivial, in fact $p=1$, and $c=1$ verifying the implication $(4.3) \Rightarrow(4.6)$.

To complete the proof, suppose now that (4.6) holds. For any $a, b \in A_{1+}^{-1}$ we infer that

$$
\sigma\left(\phi(a) \phi(b)^{-1}\right)=\sigma\left(b_{0} J(a) J(b)^{-1} b_{0}^{-1}\right)
$$

$$
=\sigma\left(b_{0} J(a) J\left(b^{-1}\right) b_{0}^{-1}\right)=\sigma\left(J(a) J\left(b^{-1}\right)\right)=\sigma\left(a b^{-1}\right)
$$

and hence we obtain (4.1).

Observe that the implication (4.3) $\Rightarrow$ (4.5) gives a substantial generalization of our former result Theorem 9 in [5] about the structure of Thompson isometries between the positive definite cones of $C^{*}$-algebras which is one of the main results in that paper. Indeed, one needs only to choose $h_{1}=h_{2}=\log$ to obtain that result from Theorem 4 .

We also remark that in [8] we have presented structural results for surjective maps between the positive definite cones of factor von Neumann algebras which respect a pair of generalized distance measures of the form similar to what appears in (4.3) above with the difference that in [8] we have considered arbitrary unitarily invariant norms in the place of the unique $C^{*}$-algebra norm $\|$.$\| (operator norm). So in a sense those results concern more general distance measures but$ in a more restricted context. Indeed, due to the (mainly algebraic) tools we have applied there, the 
results [8] have been obtained only for factor von Neumann algebras and not, like here, for general $C^{*}$-algebras. Related results in the context of matrix algebras appeared in our former paper [9].

In what follows we present several sorts of extensions of our previous theorem.

Corollary 5. Let $A_{j}$ be a $C^{*}$-algebra for $j=1,2$ and suppose that $\phi$ and $\psi$ are surjections from $A_{1+}^{-1}$ onto $A_{2+}^{-1}$. Then the following assertions are equivalent:

(5.1) $\sigma(a b)=\sigma(\phi(a) \psi(b)), \quad a, b \in A_{1+}^{-1}$;

(5.2) $r(a b-1)=r(\phi(a) \psi(b)-1), \quad a, b \in A_{1+}^{-1}$;

(5.3) there is a continuous function $h:] 0, \infty[\rightarrow \mathbb{R}$ which satisfies (c1)-(c4) and we have

$$
\left\|h\left(b^{\frac{1}{2}} a b^{\frac{1}{2}}\right)\right\|=\left\|h\left(\psi(b)^{\frac{1}{2}} \phi(a) \psi(b)^{\frac{1}{2}}\right)\right\|, \quad a, b \in A_{1+}^{-1} ;
$$

(5.4) there exists a Jordan *-isomorphism J from $A_{1}$ onto $A_{2}$ and $b_{0} \in A_{2+}^{-1}$ such that

$$
\phi(a)=b_{0} J(a) b_{0}, \psi(a)=b_{0}^{-1} J(a) b_{0}^{-1}, \quad a \in A_{1+}^{-1} .
$$

Proof. The implication (5.1) $\Rightarrow$ (5.2) is obvious, to see (5.2) $\Rightarrow$ (5.3) set $h(t)=t-1, t \in] 0, \infty[$.

Suppose that (5.3) holds. For any $a \in A_{1+}^{-1}$ we have

$$
0=\left\|h\left(a^{-\frac{1}{2}} a a^{-\frac{1}{2}}\right)\right\|=\left\|h\left(\psi\left(a^{-1}\right)^{\frac{1}{2}} \phi(a) \psi\left(a^{-1}\right)^{\frac{1}{2}}\right)\right\|
$$

which implies $\psi\left(a^{-1}\right)^{\frac{1}{2}} \phi(a) \psi\left(a^{-1}\right)^{\frac{1}{2}}=1$, i.e., $\phi(a)=\psi\left(a^{-1}\right)^{-1}$. It then follows that

$$
\left\|h\left(b^{-\frac{1}{2}} a b^{-\frac{1}{2}}\right)\right\|=\left\|h\left(\psi\left(b^{-1}\right)^{\frac{1}{2}} \phi(a) \psi\left(b^{-1}\right)^{\frac{1}{2}}\right)\right\|=\left\|h\left(\phi(b)^{-\frac{1}{2}} \phi(a) \phi(b)^{-\frac{1}{2}}\right)\right\| .
$$

Applying Theorem 4 we obtain that there is a Jordan *-isomorphism $J$ from $A_{1}$ onto $A_{2}$ and $b_{0} \in A_{2+}^{-1}$ such that

$$
\phi(a)=b_{0} J(a) b_{0}, \quad a \in A_{1+}^{-1}
$$

Moreover, we infer that

$$
\psi(a)=\phi\left(a^{-1}\right)^{-1}=b_{0}^{-1} J(a) b_{0}^{-1}, \quad a \in A_{1+}^{-1}
$$

and obtain (5.4).

Suppose now that (5.4) holds. For any $a, b \in A_{1+}^{-1}$ we calculate

$$
\sigma(\phi(a) \psi(b))=\sigma\left(b_{0} J(a) J(b) b_{0}^{-1}\right)=\sigma(J(a) J(b))=\sigma(a b) .
$$

Thus (5.1) holds and the proof is complete.

From the above statement we immediately obtain the following corollary which presents a complete description of spectrally multiplicative maps between the positive definite cones of $C^{*}$-algebras.

Corollary 6. Let $A_{j}$ be a $C^{*}$-algebra for $j=1,2$. Suppose that $\phi$ is a surjection from $A_{1+}^{-1}$ onto $A_{2+}^{-1}$. Then the following statements are equivalent:

(6.1) $\sigma(a b)=\sigma(\phi(a) \phi(b)), \quad a, b \in A_{1+}^{-1}$;

(6.2) $r(a b-1)=r(\phi(a) \phi(b)-1), \quad a, b \in A_{1+}^{-1}$;

(6.3) there is a continuous function $h:] 0, \infty[\rightarrow \mathbb{R}$ which satisfies (c1)-(c4) and we have

$$
\left\|h\left(b^{\frac{1}{2}} a b^{\frac{1}{2}}\right)\right\|=\left\|h\left(\phi(b)^{\frac{1}{2}} \phi(a) \phi(b)^{\frac{1}{2}}\right)\right\|, \quad a, b \in A_{1+}^{-1} ;
$$

(6.4) there exists a Jordan *-isomorphism J from $A_{1}$ onto $A_{2}$ such that

$$
\phi(a)=J(a), \quad a \in A_{1+}^{-1} .
$$

Proof. In the light of the proofs of the previous results, the only implication we need to verify is $(6.3) \Rightarrow(6.4)$. Assuming (6.3), by Corollary 6 there exists a Jordan *-isomorphism $J$ from $A_{1}$ onto $A_{2}$ and $b_{0} \in A_{2+}^{-1}$ such that

$$
\phi(a)=b_{0} J(a) b_{0}, \phi(a)=b_{0}^{-1} J(a) b_{0}^{-1}, \quad a \in A_{1+}^{-1} .
$$

Choosing $a=1$, it follows that $b_{0}^{2}=b_{0}^{-2}$ which implies $b_{0}=1$ and we are done. 
With some extra efforts, from Corollary 5 we can deduce the following formally even more general result on the structure of maps defined on arbitrary sets with values in the positive definite cones of $C^{*}$-algebras with a specific property closely related to spectral multiplicativity.

Theorem 7. Let $A_{j}$ be a $C^{*}$-algebra for $j=1,2$ and $F$ a non-empty set. Suppose that $\Phi_{1}$ and $\Psi_{1}$ are surjections from $F$ onto $A_{1+}^{-1}$ and that $\Phi_{2}$ and $\Psi_{2}$ are surjections from $F$ onto $A_{2+}^{-1}$. The following statements are equivalent:

(7.1) $\sigma\left(\Phi_{1}(x) \Psi_{1}(y)\right)=\sigma\left(\Phi_{2}(x) \Psi_{2}(y)\right), \quad x, y \in F$;

(7.2) $r\left(\Phi_{1}(x) \Psi_{1}(y)-1\right)=r\left(\Phi_{2}(x) \Psi_{2}(y)-1\right), \quad x, y \in F$;

(7.3) there is a continuous function $h:] 0, \infty[\rightarrow \mathbb{R}$ which satisfies (c1)-(c4) and we have

$$
\left\|h\left(\Psi_{1}(y)^{\frac{1}{2}} \Phi_{1}(x) \Psi_{1}(y)^{\frac{1}{2}}\right)\right\|=\left\|h\left(\Psi_{2}(y)^{\frac{1}{2}} \Phi_{2}(x) \Psi_{2}(y)^{\frac{1}{2}}\right)\right\|, \quad x, y \in F ;
$$

(7.4) there exists a Jordan *-isomorphism J from $A_{1}$ onto $A_{2}$ and an element $b_{0} \in A_{2+}^{-1}$ such that

$$
\Phi_{2}(x)=b_{0} J\left(\Phi_{1}(x)\right) b_{0}, \Psi_{2}(x)=b_{0}^{-1} J\left(\Psi_{1}(x)\right) b_{0}^{-1}, \quad x, y \in F .
$$

Proof. Again, in the light of the previous proofs the implications (7.1) $\Rightarrow(7.2) \Rightarrow$ (7.3) are apparent.

Suppose that (7.3) holds. To prove (7.4), we first observe that $\Phi_{1}(x)=\Phi_{1}\left(x^{\prime}\right)$ implies $\Phi_{2}(x)=$ $\Phi_{2}\left(x^{\prime}\right)$. Indeed, let $x, x^{\prime} \in F$ and assume that $\Phi_{1}(x)=\Phi_{1}\left(x^{\prime}\right)$. Since $\Psi_{1}(F)=A_{1+}^{-1}$, there exists $y \in F$ with $\Psi_{1}(y)=\Phi_{1}(x)^{-1}$. Then we have

$$
0=\left\|h\left(\Psi_{1}(y)^{\frac{1}{2}} \Phi_{1}(x) \Psi_{1}(y)^{\frac{1}{2}}\right)\right\|=\left\|h\left(\Psi_{2}(y)^{\frac{1}{2}} \Phi_{2}(x) \Psi_{2}(y)^{\frac{1}{2}}\right)\right\|
$$

implying that $\Psi_{2}(y)^{\frac{1}{2}} \Phi_{2}(x) \Psi_{2}(y)^{\frac{1}{2}}=1$. Thus we have $\Psi_{2}(y)^{-1}=\Phi_{2}(x)$. In a similar way we obtain that $\Psi_{2}(y)^{-1}=\Phi_{2}\left(x^{\prime}\right)$ holds, too. It then follows that $\Phi_{2}(x)=\Phi_{2}\left(x^{\prime}\right)$. In the same way one can deduce that $\Psi_{1}(x)=\Psi_{1}\left(x^{\prime}\right)$ implies $\Psi_{2}(x)=\Psi_{2}\left(x^{\prime}\right)$. After this we define maps $\phi, \psi: A_{1+}^{-1} \rightarrow A_{2+}^{-1}$ by $\phi\left(\Phi_{1}(x)\right)=\Phi_{2}(x), x \in F$ and by $\psi\left(\Psi_{1}(x)\right)=\Psi_{2}(x), x \in F$. Apparently, $\phi, \psi$ are well defined and surjective. Rewriting the displayed equality in (7.3) we have

$$
\left\|h\left(b^{\frac{1}{2}} a b^{\frac{1}{2}}\right)\right\|=\left\|h\left(\psi(b)^{\frac{1}{2}} \phi(a) \psi(b)^{\frac{1}{2}}\right)\right\|, \quad a, b \in A_{1+}^{-1} .
$$

By Corollary 5 there exists a Jordan *-isomorphism $J$ from $A_{1}$ onto $A_{2}$ and an element $b_{0} \in A_{2+}^{-1}$ such that

In other words, we have

$$
\phi(a)=b_{0} J(a) b_{0}, \psi(a)=b_{0}^{-1} J(a) b_{0}^{-1}, \quad a \in A_{1+}^{-1} .
$$

$$
\Phi_{2}(x)=b_{0} J\left(\Phi_{1}(x)\right) b_{0}, \Psi_{2}(x)=b_{0}^{-1} J\left(\Psi_{1}(x)\right) b_{0}^{-1}, \quad x \in F
$$

and this proves (7.4).

Finally, in a way similar to the proof of Corollary 5 one can check that (7.4) implies (7.1) which finishes the proof of the theorem.

We conclude the section with a few other corollaries which provide characterizations of Jordan *-isomorphisms of the self-adjoint parts of $C^{*}$-algebras by means of their certain spectral multiplicativity type properties.

Corollary 8. Let $A_{j}$ be a $C^{*}$-algebra for $j=1,2$. Suppose that $f$ and $g$ are surjections from $A_{1 s}$ onto $A_{2 s}$. Then the following assertions are equivalent:

(8.1) $\sigma(\exp x \exp y)=\sigma(\exp f(x) \exp g(y)), \quad x, y \in A_{1 s}$;

(8.2) $r(\exp x \exp y-1)=r(\exp f(x) \exp g(y)-1), \quad x, y \in A_{1 s}$;

(8.3) there is a continuous function $h:] 0, \infty[\rightarrow \mathbb{R}$ which satisfies (c1)-(c4) and we have

$\|h(\exp (y / 2) \exp (x) \exp (y / 2))\|$

$$
=\|h(\exp (g(y) / 2) \exp (f(x)) \exp (g(y) / 2))\|, \quad x, y \in A_{1 s} ;
$$

(8.4) there exists a Jordan *-isomorphism J from $A_{1 s}$ onto $A_{2 s}$ and $b_{0} \in A_{2+}^{-1}$ such that

$$
\exp f(x)=b_{0}(\exp J(x)) b_{0}, \exp g(x)=b_{0}^{-1}(\exp J(x)) b_{0}^{-1}, \quad x, y \in A_{1 s} .
$$

Moreover, in any of the above cases, if $f(0)=0$, then we $f=g=J$ on $A_{1 s}$. 
Proof. Define $\phi(a)=\exp (f(\log a)), a \in A_{1+}^{-1}$ and $\psi(b)=\exp (g(\log b)), b \in A_{1+}^{-1}$. Apply Corollary 5 to see the equivalence of the assertions (8.1)-(8.4). If $f(0)=0$, we easily obtain $b_{0}=1$ which implies $f=g=J$ on $A_{1 s}$.

If we have $f=g$ in the previous corollary, we trivially obtain the following statement.

Corollary 9. Let $A_{j}$ be a $C^{*}$-algebra for $j=1,2$. Suppose that $f$ is a surjection from $A_{1 s}$ onto $A_{2 s}$. Then the following assertions are equivalent:

(9.1) $\sigma(\exp x \exp y)=\sigma(\exp f(x) \exp f(y)), \quad x, y \in A_{1 s}$;

(9.2) $r(\exp x \exp y-1)=r(\exp f(x) \exp f(y)-1), \quad x, y \in A_{1 s}$;

(9.3) there is a continuous function $h:] 0, \infty[\rightarrow \mathbb{R}$ which satisfies (c1)-(c4) and we have

$\|h(\exp (y / 2) \exp (x) \exp (y / 2))\|$

$$
=\|h(\exp (f(y) / 2) \exp (f(x)) \exp (f(y) / 2))\|, \quad x, y \in A_{1 s} ;
$$

(9.4) there exists a Jordan *-isomorphism $J$ from $A_{1 s}$ onto $A_{2 s}$ such that $f=J$ on $A_{1 s}$.

Similarly, putting $g(y)=-f(-y), y \in \mathscr{A}_{1 s}$ into Corollary 8 we have the following statement.

Corollary 10. Let $A_{j}$ be a $C^{*}$-algebra for $j=1,2$. Suppose that $f$ is a surjection from $A_{1 s}$ onto $A_{2 s}$. Then the following assertions are equivalent:

(10.1) $\sigma\left(\exp x(\exp y)^{-1}\right)=\sigma\left(\exp f(x)(\exp f(y))^{-1}\right), \quad x, y \in A_{1 s}$;

(10.2) $r\left(\exp x(\exp y)^{-1}-1\right)=r\left(\exp f(x)(\exp f(y))^{-1}-1\right), \quad x, y \in A_{1 s}$;

(10.3) there is a continuous function $h:] 0, \infty[\rightarrow \mathbb{R}$ which satisfies (c1)-(c4) and we have

$$
\left\|h\left(\exp (y)^{-\frac{1}{2}} \exp (x) \exp (y)^{-\frac{1}{2}}\right)\right\|
$$

$$
=\left\|h\left(\exp (f(y))^{-\frac{1}{2}} \exp (f(x)) \exp (f(y))^{-\frac{1}{2}}\right)\right\|, \quad x, y \in A_{1 s}
$$

(10.4) there exists a Jordan *-isomorphism J from $A_{1 s}$ onto $A_{2 s}$ and $b_{0} \in A_{2+}^{-1}$ such that

$$
\exp f(x)=b_{0}(\exp J(x)) b_{0}, \quad x, y \in A_{1 s} .
$$

Moreover, in any of the above cases, if $f(0)=0$, then $f=J$ holds on $A_{1 s}$.

\section{THE CASE OF THE UNITARY GROUPS}

In the last part of our paper we present spectral conditions for Jordan *-isomorphisms between the unitary groups of von Neumann algebras. In the proof of our second main result Theorem 12 below we apply the next general Mazur-Ulam type result relating groups. It appeared as Proposition 20 in [8] (cf. Corollary 3.9 in [3]).

Theorem 11. Suppose that $G$ and $H$ are groups equipped with generalized distance measures $d$ and $\rho$, respectively. Pick $a, b \in G$, set

$$
L_{a, b}=\left\{x \in G: d(a, x)=d\left(x, b a^{-1} b\right)=d(a, b)\right\},
$$

and assume the following:

(d1) $d\left(b x^{-1} b, b x^{\prime-1} b\right)=d\left(x^{\prime}, x\right)$ holds for all $x, x^{\prime} \in G$;

(d2) $\sup \left\{d(x, b): x \in L_{a, b}\right\}<\infty$;

(d3) there exists a constant $K>1$ such that

$$
d\left(x, b x^{-1} b\right) \geq K d(x, b), \quad x \in L_{a, b} ;
$$

(d4) $\rho\left(c y^{-1} c^{\prime}, c y^{\prime-1} c^{\prime}\right)=\rho\left(y^{\prime}, y\right)$ holds for all $c, c^{\prime}, y, y^{\prime} \in H$.

Then for any surjective map $\phi: G \rightarrow H$ which satisfies

$$
\rho\left(\phi(x), \phi\left(x^{\prime}\right)\right)=d\left(x, x^{\prime}\right), \quad x, x^{\prime} \in G
$$

we have

$$
\phi\left(b a^{-1} b\right)=\phi(b) \phi(a)^{-1} \phi(b) .
$$


One may ask if the above statement can be deduced from Theorem 3. The easy answer is "no", since the natural operation $a b^{-1} a$ called the inverted Jordan triple product in a group does not generally satisfy the uniqueness part of the condition in (a3).

Analogously to Section 2, below we shall consider generalized distance measures on unitary groups obtained from continuous functions defined on the unit circle $\mathbb{T}$.

For any continuous function $h: \mathbb{T} \rightarrow \mathbb{C}$ we shall consider the following properties:

(e1) $h(z)=0$ holds exactly for $z=1$;

(e2) $h$ is differentiable at $z=1$ meaning that the $\operatorname{limit}_{z \rightarrow 1} \frac{h(z)-h(1)}{z-1}$ exists and we assume that it is non-zero.

Observe that just as in the remark (R4) one can prove that the condition imply that for any number $0 \leq K<2$ we have $\left|h\left(z^{2}\right)\right| \geq K|h(z)|$ for all $z \in \mathbb{T}$ close enough to 1 .

The second main result of the paper reads as follows.

Theorem 12. Let $M_{j}$ be a von Neumann algebra with unitary group $U_{j}$ for $j=1,2$. Suppose that $\phi$ is a surjection from $U_{1}$ onto $U_{2}$. The following two conditions are equivalent:

(12.1) $\sigma\left(a b^{-1}\right)=\sigma\left(\phi(a) \phi(b)^{-1}\right), \quad a, b \in U_{1}$;

(12.2) there exists a Jordan *-isomorphism J from $M_{1}$ onto $M_{2}$ and an element $u_{0} \in U_{2}$ such that

$$
\phi(a)=u_{0} J(a), \quad a \in U_{1} .
$$

Moreover, the following three conditions are also equivalent:

(12.3) $r\left(a b^{-1}-1\right)=r\left(\phi(a) \phi(b)^{-1}-1\right), \quad a, b \in U_{1}$;

(12.4) there exists a pair $h_{1}, h_{2}: \mathbb{T} \rightarrow \mathbb{C}$ of continuous functions which satisfy (e1)-(e2) and we have

$$
\left\|h_{1}\left(a b^{-1}\right)\right\|=\left\|h_{2}\left(\phi(a) \phi(b)^{-1}\right)\right\|, \quad a, b \in U_{1} ;
$$

(12.5) there exists a Jordan *-isomorphism J from $M_{1}$ onto $M_{2}$, an element $u_{0} \in U_{2}$ and a central projection $p \in A_{2}$ such that

$$
\phi(a)=u_{0}\left(p J(a)+(1-p) J(a)^{-1}\right), \quad a \in U_{1} .
$$

Proof. We begin with the second part of the theorem. To see the implication (12.3) $\Rightarrow$ (12.4) consider simply the functions $h_{1}(z)=h_{2}(z)=z-1, z \in \mathbb{T}$.

In the rest of the proof we follow the proof of Theorem 4 rather closely in spirit. Assume that (12.4) holds with continuous functions $h_{1}, h_{2}: \mathbb{T} \rightarrow \mathbb{C}$ satisfying (e1)-(e2). Define

$$
d(a, b)=\left\|h_{1}\left(a b^{-1}\right)\right\|, \quad a, b \in U_{1}
$$

and

$$
\rho(a, b)=\left\|h_{2}\left(a b^{-1}\right)\right\|, \quad a, b \in U_{2} .
$$

By the property (e1) we obtain that $d, \rho$ are generalized distance measures and we have

$$
\rho(\phi(a), \phi(b))=d(a, b), \quad a, b \in U_{1} .
$$

One can easily check that

$$
d(z a w, z b w)=d(a, b)
$$

and

$$
d\left(b x^{-1} b, b x^{\prime-1} b\right)=d\left(x^{-1}, x^{\prime-1}\right)=d\left(x^{\prime}, x\right)
$$

are satisfied for all $a, b, x, z, w \in U_{1}$. Clearly, similar properties hold for the generalized distance measure $\rho$, too.

Define the map $\phi_{0}: U_{1} \rightarrow U_{2}$ by $\phi_{0}(a)=\phi(1)^{-1} \phi(a), a \in U_{1}$. Plainly, $\phi_{0}$ is a well defined and surjective map from $U_{1}$ onto $U_{2}$, it is unital meaning that $\phi_{0}(1)=1$, and the equality (7) holds also for $\phi_{0}$, i.e., we have

$$
\rho\left(\phi_{0}(a), \phi_{0}(b)\right)=d(a, b), \quad a, b \in U_{1} .
$$

We are going to apply Theorem 11 for $G=U_{1}, H=U_{2}$, for the above defined distance measures $d, \rho$ and for the surjective map $\phi_{0}$. We have seen in (8) that the conditions (d1), (d4) in Theorem 11 
are satisfied. The condition (d2) is also fulfilled as a consequence of the boundedness of the continuous function $h_{1}$. Now we show that (d3) is satisfied for such $a, b \in U_{1}$ which are close enough to each other in norm. To see this, we shall need the following simple observation: for any sequences $a_{n}, b_{n}$ in $U_{1}$ we have

$$
\left\|a_{n}-b_{n}\right\|=\left\|a_{n} b_{n}^{-1}-1\right\| \rightarrow 0 \Leftrightarrow\left\|h_{1}\left(a_{n} b_{n}^{-1}\right)\right\|=d\left(a_{n}, b_{n}\right) \rightarrow 0
$$

and similar observation holds for the generalized distance measure $\rho$ as well. In fact, this follows rather easily from the continuity of $h_{1}$ and the property (e1). In particular, we obtain that "convergence" in any of the generalized distance measures $d, \rho$ is equivalent to the convergence in the norm $\|$.$\| .$

In order to show that the condition (d3) holds for close enough $a, b \in U_{1}$, assume on the contrary that we have sequences $a_{n}, b_{n} \in U_{1}$ and $x_{n} \in L_{a_{n}, b_{n}}$ such that $\left\|a_{n}-b_{n}\right\| \rightarrow 0$ and

$$
d\left(x_{n}, b_{n} x_{n}^{-1} b_{n}\right)<(3 / 2) d\left(x_{n}, b_{n}\right)
$$

holds for every positive integer $n$. This latter inequality means that

$$
\left\|h_{1}\left(\left(x_{n} b_{n}^{-1}\right)^{2}\right)\right\|<(3 / 2)\left\|h_{1}\left(x_{n} b_{n}^{-1}\right)\right\|
$$

holds for all $n$. Since $d\left(a_{n}, x_{n}\right)=d\left(a_{n}, b_{n}\right) \rightarrow 0$, we have $a_{n} x_{n}^{-1}, a_{n} b_{n}^{-1} \rightarrow 1$ in norm which apparently implies that $x_{n} b_{n}^{-1} \rightarrow 1$ in norm. On the other hand, we know that $\left|h\left(z^{2}\right)\right| \geq(3 / 2)|h(z)|$ for all $z \in \mathbb{T}$ which are close enough to 1 . Therefore, we obtain $\left\|h_{1}\left(\left(x_{n} b_{n}^{-1}\right)^{2}\right)\right\| \geq 3 / 2\left\|h_{1}\left(x_{n} b_{n}^{-1}\right)\right\|$ for large enough $n$, a contradiction. This shows that the condition (d3) is satisfied for close enough $a, b \in U_{1}$. Applying Theorem 11 it follows that there is $\delta>0$ such that for every $\|a-b\|<\delta, a, b \in U_{1}$ we have

$$
\phi_{0}\left(b a^{-1} b\right)=\phi_{0}(b) \phi_{0}(a)^{-1} \phi_{0}(b) .
$$

Just as in the first section of the proof of Theorem 1 in [5] we then deduce that

$$
\phi_{0}\left(b a^{-1} b\right)=\phi_{0}(b) \phi_{0}(a)^{-1} \phi_{0}(b)
$$

holds not only locally, but also globally, i.e., for any $a, b \in U_{1}$. Putting $b=1$ we get $\phi_{0}\left(a^{-1}\right)=\phi_{0}(a)^{-1}$ for every $a \in U_{1}$ and then obtain that

$$
\phi_{0}(b a b)=\phi_{0}(b) \phi_{0}(a) \phi_{0}(b), \quad a, b \in U_{1} .
$$

By the equivalence of the convergence in $d, \rho$ and in the norm we deduce that $\phi_{0}$ is norm-continuous. Therefore, following the proof of Theorem 1 in [5] on page 160-161 employing one-parameter unitary groups, we infer that there is a bijective map $f: M_{1 s} \rightarrow M_{2 s}$ with $f(0)=0$ for which

$$
\phi_{0}(\exp (i t x))=\exp (i t f(x)), \quad t \in \mathbb{R}, x \in M_{1 s} .
$$

Just as in the proof of Theorem 4 we claim that $f$ is a scalar multiple of a norm-isometry. To verify this, we observe that one can prove similarly to (5) that

$$
\frac{d(\exp (i t x), \exp (\text { it } y))}{|t|} \rightarrow\left|h_{1}^{\prime}(1)\right|\|x-y\|
$$

holds for all $x, y \in M_{1 s}$ as $t \rightarrow 0$. We omit the details. Similarly, we obtain that

$$
\frac{\rho(\exp (i t x), \exp (i t y))}{|t|} \rightarrow\left|h_{2}^{\prime}(1)\right|\|x-y\|
$$

holds for all $x, y \in M_{2 s}$ as $t \rightarrow 0$. Since $\phi_{0}$ respects the pair $d, \rho$ of generalized distance measures, i.e., satisfies (9), it follows that $\left|h_{1}^{\prime}(1)\right|\|x-y\|=\left|h_{2}^{\prime}(1)\right|\|f(x)-f(y)\|, x, y \in M_{1 s}$. This implies that we have a positive scalar $c$ such that $(1 / c) f$ is a surjective isometry from $M_{1 s}$ onto $M_{2 s}$. Just as in the proof of Theorem 4, since $f(0)=0$, by Mazur-Ulam theorem we infer that $f$ is linear and next apply Kadison's theorem [6, Therem 2] to obtain that $(1 / c) f(1)$ is a central symmetry in $M_{2}$ and there is a Jordan *-isomorphism $J$ from $M_{1}$ onto $M_{2}$ such that

$$
f(x)=f(1) J(x), \quad x \in M_{1 s} .
$$

Set $p=(1+(1 / c) f(1)) / 2$. Then $p$ is a central projection in $M_{2}$ and

$$
f(x)=c(p J(x)-(1-p) J(x)), \quad x \in M_{1 s} .
$$


Next an easy calculation yields that

$$
\phi_{0}(\exp i x)=\exp (c(p J(i x)-(1-p) J(i x)))
$$

$$
=p J(\exp (i c x))+(1-p) J(\exp (-i c x))
$$

holds for every $x \in M_{1 s}$. We assert that $c$ is necessarily an integer. Indeed, since $\phi_{0}$ is unital and satisfies (10), it follows that $\phi_{0}$ sends symmetries to symmetries. Therefore, for any non-zero projection $q$ in $M_{1}$, the spectrum of

$$
\phi_{0}(\exp i \pi q)=p J(\exp (i c \pi q))+(1-p) J(\exp (-i c \pi q))
$$

is contained in $\{-1,1\}$. Since $J$ preserves the spectrum and $p$ is central, it follows that at least one of the sets $\{1, \exp (i c \pi)\},\{1, \exp (-i c \pi)\}$ ( $p$ might be trivial) is contained in $\{-1,1\}$. This gives as that $c$ is an integer and since it is assumed also to be positive, we obtain that $c$ is a positive integer. Therefore, by (11) we have

$$
\phi_{0}(a)=p J\left(a^{c}\right)+(1-p) J\left(a^{-c}\right), \quad a \in U_{1} .
$$

Now we prove that $c=1$. Indeed, assuming that the central projection $p$ above is non-trivial, inserting scalars $a=z 1, z \in \mathbb{T}$ and $b=1$ into (12), and using the generalized distance measure preserving property of $\phi_{0}$, we easily obtain

$$
\left|h_{1}(z)\right|=\max \left\{\left|h_{2}\left(z^{c}\right)\right|,\left|h_{2}\left(z^{-c}\right)\right|\right\}
$$

for all $z \in \mathbb{T}$. Since $h_{1}, h_{2}$ has unique roots at $z=1$, we infer that $c$ must be 1 . Similar argument works also in the case where $p$ is trivial. This completes the proof of the implication (12.4) $\Rightarrow(12.5)$.

Assume (12.5) holds. We compute

$$
\begin{aligned}
r\left(\phi(a) \phi(b)^{-1}-1\right)= & \left\|\phi(a) \phi(b)^{-1}-1\right\| \\
= & \left\|u_{0}\left(p J(a) J(b)^{-1}+(1-p) J(a)^{-1} J(b)\right) u_{0}^{-1}-1\right\| \\
=\left\|p J(a) J(b)^{-1}+(1-p) J(a)^{-1} J(b)-1\right\| & =\max \left\{\left\|p\left(J(a) J(b)^{-1}-1\right)\right\|,\left\|(1-p)\left(J(a)^{-1} J(b)-1\right)\right\|\right\} .
\end{aligned}
$$

Furthermore, by taking adjoints we have

$$
\begin{aligned}
& \left\|(1-p)\left(J(a)^{-1} J(b)-1\right)\right\|=\left\|(1-p)\left(J(b)^{-1} J(a)-1\right)\right\| \\
& \quad=\left\|J(b)^{-1}(1-p)(J(a)-J(b))\right\|=\left\|(1-p)(J(a)-J(b)) J(b)^{-1}\right\| \\
& =\left\|(1-p)\left(J(a) J(b)^{-1}-1\right)\right\|
\end{aligned}
$$

since $1-p$ commutes with every element in $M_{2}$. It follows from (13) and (14) that

$$
\begin{aligned}
& r\left(\phi(a) \phi(b)^{-1}-1\right)=\max \left\{\left\|p\left(J(a) J(b)^{-1}-1\right)\right\|,\left\|(1-p)\left(J(a) J(b)^{-1}-1\right)\right\|\right\} \\
&=\left\|p\left(J(a) J(b)^{-1}-1\right)+(1-p)\left(J(a) J(b)^{-1}-1\right)\right\|=\left\|J(a) J(b)^{-1}-1\right\| \\
&=r\left(J(a) J\left(b^{-1}\right)-1\right)=r\left(a b^{-1}-1\right) .
\end{aligned}
$$

The last equality follows from the spectral multiplicativity of $J$. Thus we obtain (12.3).

Let us consider now the first part of the theorem. Assume (12.1) holds. It trivially implies (12.3) which implies (12.5). Consequently, there exists a Jordan *-isomorphism $J$ from $M_{1}$ onto $M_{2}$, an element $u_{0} \in U_{2}$ and a central projection $p \in A_{2}$ such that

$$
\phi(a)=u_{0}\left(p J(a)+(1-p) J(a)^{-1}\right), \quad a \in U_{1} ;
$$

It is not hard to verify that the central projection $p$ above must be the identity and hence we obtain (12.2). The implication (12.2) $\Rightarrow(12.1)$ is trivial to check and hence the proof of the theorem is complete.

Corollary 13. Let $M_{j}$ be a von Neumann algebra with unitary group $U_{j}$ for $j=1,2$. Suppose that $\phi$ and $\psi$ are surjections from $U_{1}$ onto $U_{2}$. Then the following two conditions are equivalent: 
(13.1) $\sigma(a b)=\sigma(\phi(a) \psi(b)), \quad a, b \in U_{1}$;

(13.2) there exists a Jordan *-isomorphism J from $M_{1}$ onto $M_{2}$ and $u_{0} \in U_{2}$ such that

$$
\phi(a)=u_{0} J(a), \psi(a)=J(a) u_{0}^{-1}, \quad a \in U_{1} .
$$

Moreover the following three conditions are also equivalent:

(13.3) $r(a b-1)=r(\phi(a) \psi(b)-1), \quad a, b \in U_{1}$;

(13.4) there exists a pair $h_{1}, h_{2}: \mathbb{T} \rightarrow \mathbb{C}$ of continuous functions which satisfy (e1)-(e2) and we have

$$
\left\|h_{1}(a b)\right\|=\left\|h_{2}(\phi(a) \psi(b))\right\|, \quad a, b \in U_{1} ;
$$

(13.5) there exists a Jordan *-isomorphism $J$ from $M_{1}$ onto $M_{2}$, a central projection $p \in M_{2}$, and $u_{0} \in U_{2}$ such that

$$
\phi(a)=u_{0}\left(p J(a)+(1-p) J(a)^{-1}\right), \psi(a)=\left(p J(a)+(1-p) J(a)^{-1}\right) u_{0}^{-1}, \quad a \in U_{1} .
$$

Proof. Setting $b=a^{-1}$, from both of (13.1) and (13.4) we obtain $\psi\left(a^{-1}\right)=\phi(a)^{-1}$. Easy application of Theorem 12 gives the implications (13.1) $\Rightarrow$ (13.2) and (13.4) $\Rightarrow$ (13.5). The rest of the proof can be shown by arguments already employed in the previous part of the paper. For example, the implication (13.5) $\Rightarrow$ (13.3) can be proved by a reasoning similar to the one that has appeared in the proof of the implication (12.5) $\Rightarrow$ (12.3). We omit the details.

Corollary 14. Let $M_{j}$ be a von Neumann algebra with unitary group $U_{j}$ for $j=1,2$. Suppose that $\phi$ is a surjection from $U_{1}$ onto $U_{2}$. Then the following conditions are equivalent:

(14.1) $\sigma(a b)=\sigma(\phi(a) \phi(b)), \quad a, b \in U_{1}$;

(14.2) there exists a Jordan *-isomorphism J from $M_{1}$ onto $M_{2}$ and a central symmetry $u_{0} \in U_{2}$ such that

$$
\phi(a)=u_{0} J(a), \quad a \in U_{1} .
$$

Moreover the following three conditions are also equivalent:

(14.3) $r(a b-1)=r(\phi(a) \phi(b)-1), \quad a, b \in U_{1}$;

(14.4) there exists a pair $h_{1}, h_{2}: \mathbb{T} \rightarrow \mathbb{C}$ of continuous functions which satisfy (e1)-(e2) and we have

$$
\left\|h_{1}(a b)\right\|=\left\|h_{2}(\phi(a) \phi(b))\right\|, \quad a, b \in U_{1} ;
$$

(14.5) there exists a Jordan *-isomorphism $J$ from $M_{1}$ onto $M_{2}$, a central projection $p \in M_{2}$, and a central symmetry $u_{0} \in U_{2}$ such that

$$
\phi(a)=u_{0}\left(p J(a)+(1-p) J(a)^{-1}\right), \quad a \in U_{1} .
$$

Proof. We apply Theorem 13 for $\psi=\phi$. The only implications that need closer look are (14.1) $\Rightarrow$ (14.2) and (14.4) $\Rightarrow$ (14.5). Assuming (14.1) we have a Jordan ${ }^{*}$-isomorphism $J: M_{1} \rightarrow M_{2}$ and an element $u_{0} \in U_{2}$ such that $\phi(a)=u_{0} J(a)=J(a) u_{0}^{-1}, a \in U_{1}$. Since the unitary group linearly generate the whole algebra, it follows that $u_{0} x=x u_{0}^{-1}$ holds for all $x \in M_{2}$ which readily implies that $u_{0}=u_{0}^{-1}$ and then that $u_{0}$ is central. Similar argument applies for the implication (14.4) $\Rightarrow$ (14.5), the rest of the proof is either easy or can be proved by already employed arguments.

In order to avoid overcomplications in the formulations of the remaining results, in what follows we shall omit conditions regarding the invariance properties of the transformations under considerations with respect to generalized distance measures. We are convinced that having read the paper carefully up to this point, it would be an easy task for the reader to complete the results with such additional equivalent conditions.

Theorem 15. Let $M_{j}$ be a von Neumann algebra with unitary group $U_{j}$ for $j=1,2$ and $F$ a nonempty set. Suppose that $\Phi_{j}$ and $\Psi_{j}$ are surjections from $F$ onto $U_{j}$ for $j=1,2$. Then the following two conditions are equivalent

(15.1) $\sigma\left(\Phi_{1}(x) \Psi_{1}(y)\right)=\sigma\left(\Phi_{2}(x) \Psi_{2}(y)\right), \quad x, y \in F$;

(15.2) there exists a Jordan *-isomorphism J from $M_{1}$ onto $M_{2}$ and $u_{0} \in U_{2}$ such that

$$
\Phi_{2}(x)=u_{0} J\left(\Phi_{1}(x)\right), \Psi_{2}(x)=J\left(\Psi_{1}(x)\right) u_{0}^{-1}, \quad x \in F .
$$


Moreover, the following two conditions are also equivalent:

(15.3) $r\left(\Phi_{1}(x) \Psi_{1}(y)-1\right)=r\left(\Phi_{2}(x) \Psi_{2}(y)-1\right), \quad x, y \in F$;

(15.4) there exists a Jordan *-isomorphism $J$ from $M_{1}$ onto $M_{2}$, a central projection $p \in M_{2}$, and $u_{0} \in U_{2}$ such that

$$
\Phi_{2}(x)=u_{0}\left(p J\left(\Phi_{1}(x)\right)+(1-p) J\left(\Phi_{1}(x)\right)^{-1}\right), \quad x \in F
$$

and

$$
\Psi_{2}(x)=\left(p J\left(\Psi_{1}(x)\right)+(1-p) J\left(\Psi_{1}(x)\right)^{-1}\right) u_{0}^{-1}, \quad x \in F .
$$

Proof. Suppose that (15.2) holds. We simply infer that

$$
\sigma\left(\Phi_{2}(x) \Psi_{2}(y)\right)=\sigma\left(J\left(\Phi_{1}(x)\right) J\left(\Psi_{1}(y)\right)\right)=\sigma\left(\Phi_{1}(x) \Psi_{1}(y)\right), \quad x, y \in F .
$$

Suppose now that (15.1) holds. We first observe that $\Phi_{1}(x)=\Phi_{1}\left(x^{\prime}\right)$ implies that $\Phi_{2}(x)=\Phi_{2}\left(x^{\prime}\right)$. Indeed, assume that $\Phi_{1}(x)=\Phi_{1}\left(x^{\prime}\right)$. Then we obtain

$$
\sigma\left(\Phi_{2}(x) \Psi_{2}(y)\right)=\sigma\left(\Phi_{1}(x) \Psi_{1}(y)\right)=\sigma\left(\Phi_{1}\left(x^{\prime}\right) \Psi_{1}(y)\right)=\sigma\left(\Phi_{2}\left(x^{\prime}\right) \Psi_{2}(y)\right), \quad y \in F .
$$

Pick $y \in F$ with $\Psi_{2}(y)=\Phi_{2}(x)^{-1}$. Such an element $y \in F$ exists since $\Psi_{2}(F)=U_{2}$. With this $y$ we have that

$$
\{1\}=\sigma\left(\Phi_{2}(x) \Psi_{2}(y)\right)=\sigma\left(\Phi_{2}\left(x^{\prime}\right) \Psi_{2}(y)\right) .
$$

From this we infer that $1=\Phi_{2}\left(x^{\prime}\right) \Psi_{2}(y)$, thus we have

$$
\Phi_{2}\left(x^{\prime}\right)=\Psi_{2}(y)^{-1}=\Phi_{2}(x) .
$$

In the same way we see that $\Psi_{1}(x)=\Psi_{1}\left(x^{\prime}\right)$ implies that $\Psi_{2}(x)=\Psi_{2}\left(x^{\prime}\right)$. Define maps $\phi, \psi: U_{1} \rightarrow$ $U_{2}$ by $\phi\left(\Phi_{1}(x)\right)=\Phi_{2}(x)$ and $\psi\left(\Psi_{1}(x)\right)=\Psi_{2}(x), x \in F$. Clearly, $\phi, \psi$ are well defined surjections from $U_{1}$ onto $U_{2}$. Moreover, we have

$$
\sigma(a b)=\sigma(\phi(a) \psi(b)), \quad a, b \in U_{1} .
$$

By Theorem 13 there exists a Jordan *-isomorphism from $M_{1}$ onto $M_{2}$ and $u_{0} \in U_{2}$ such that

$$
\phi(a)=u_{0} J(a), \psi(a)=J(a) u_{0}^{-1}, \quad a \in U_{1}
$$

and we easily conclude that (15.2) holds.

The implication (15.4) $\Rightarrow$ (15.3) can be proved by a reasoning similar to the one that has appeared in the proof of the implication (12.5) $\Rightarrow$ (12.3).

Suppose now that (15.3) holds. We first observe that $\Phi_{1}(x)=\Phi_{1}\left(x^{\prime}\right)$ implies that $\Phi_{2}(x)=\Phi_{2}\left(x^{\prime}\right)$ for any $x, x^{\prime} \in F$. Indeed, assume $\Phi_{1}(x)=\Phi_{1}\left(x^{\prime}\right)$. Then we have

$$
r\left(\Phi_{2}(x) \Psi_{2}(y)-1\right)=r\left(\Phi_{1}(x) \Psi_{1}(y)-1\right)=r\left(\Phi_{1}\left(x^{\prime}\right) \Psi_{1}(y)-1\right)
$$

$$
=r\left(\Phi_{2}\left(x^{\prime}\right) \Psi_{2}(y)-1\right), \quad y \in F .
$$

As $\Psi_{2}(F)=U_{2}$, there exists $y \in F$ with $\Psi_{2}(y)=\Phi_{2}(x)^{-1}$. With this $y$ we obtain

$$
0=r\left(\Phi_{2}(x) \Psi_{2}(y)-1\right)=r\left(\Phi_{2}\left(x^{\prime}\right) \Psi_{2}(y)-1\right) .
$$

As $\Phi_{2}\left(x^{\prime}\right) \Psi_{2}(y)$ is unitary, we have

$$
\left\|\Phi_{2}\left(x^{\prime}\right) \Psi_{2}(y)-1\right\|=r\left(\Phi_{2}\left(x^{\prime}\right) \Psi_{2}(y)-1\right)=0
$$

implying

$$
\Phi_{2}\left(x^{\prime}\right)=\Psi_{2}(y)^{-1}=\Phi_{2}(x) .
$$

In a similar way we see that $\Psi_{1}(x)=\Psi_{1}\left(x^{\prime}\right)$ implies $\Psi_{2}(x)=\Psi_{2}\left(x^{\prime}\right)$. Once again, define maps $\phi, \psi: U_{1} \rightarrow U_{2}$ by $\phi\left(\Phi_{1}(x)\right)=\Phi_{2}(x)$ and $\psi\left(\Psi_{1}(x)\right)=\Psi_{2}(x), x \in F$ which turn to be well defined and surjective. Moreover, we infer that

$$
r(a b-1)=r(\phi(a) \psi(b)-1), \quad a, b \in U_{1} .
$$

Then by Theorem 13 there exists a Jordan *-isomorphism, a central projection $p \in M_{2}$ and $u_{0} \in U_{2}$ such that

$$
\phi(a)=u_{0}\left(p J(a)+(1-p) J(a)^{-1}\right), \psi(a)=\left(p J(a)+(1-p) J(a)^{-1}\right) u_{0}^{-1}, \quad a \in U_{1} .
$$


This apparently gives us that (15.4) holds.

Finally, we present corollaries of the former results from which non-linear spectral multiplicativity type conditions can be deduced for maps between the self-adjoint parts of von Neumann algebras to be Jordan *-isomorphisms.

Corollary 16. Let $M_{j}$ be a von Neumann algebra for $j=1,2$. Suppose that $f$ and $g$ are bijections from $M_{1 s}$ onto $M_{2 s}$. Then the following two conditions are equivalent:

(16.1) $\sigma(\exp (i x) \exp (i y))=\sigma(\exp (i f(x)) \exp (i g(y))), \quad x, y \in M_{1 s}$;

(16.2) there exists a Jordan *-isomorphism J from $M_{1}$ onto $M_{2}$ and $u_{0} \in U_{2}$ such that

$$
\exp (i f(x))=u_{0} \exp (i J(x)), \exp (i g(x))=(\exp (i J(x))) u_{0}^{-1}, \quad x \in M_{1 s} .
$$

In particular, if $f$ and $g$ are homogeneous, then we have $f=g=J$ and $u_{0}=1$.

Moreover, the following two conditions are also equivalent:

(16.3) $r(\exp (i x) \exp (i y)-1)=r(\exp (i f(x)) \exp (i g(y))-1), \quad x, y \in M_{1 s}$;

(16.4) there exists a Jordan *-isomorphism $J$ from $M_{1}$ onto $M_{2}$, a central projection $p \in M_{2}$ and $u_{0} \in U_{2}$ such that

$$
\exp (i f(x))=u_{0}\left(p \exp (i J(x))+(1-p)(\exp (i J(x)))^{-1}\right)
$$

and

$$
\exp (i g(x))=\left(p \exp (i J(x))+(1-p)(\exp (i J(x)))^{-1}\right) u_{0}^{-1}
$$

for every $x \in M_{1 s}$.

In particular, if $f$ and $g$ are homogeneous, then we have $f=g=(2 p-1) J$ and $u_{0}=1$.

Proof. Suppose that (16.2) holds. We infer that

$$
\begin{aligned}
\sigma(\exp (i f(x)) \exp (i g(y)))=\sigma\left(u_{0} \exp (i J(x)) \exp (i J(y)) u_{0}^{-1}\right) & \\
& =\sigma(J(\exp (i x)) J(\exp (i y)))=\sigma(\exp (i x) \exp (i y)), \quad x, y \in M_{1 s} .
\end{aligned}
$$

In particular, if $f$ is homogeneous, then $f(0)=0$. It follows that $u_{0}=1$ and we have

$$
\exp (i t f(x))=\exp (i f(t x))=\exp (i t J(x)), \quad t \in \mathbb{R}, x \in M_{1 s} .
$$

Letting $t \rightarrow 0$, from

$$
(\exp (i t f(x))-1) / t=(\exp (i t J(x))-1) / t
$$

we obtain $f(x)=J(x), x \in M_{1 s}$. In the same way we deduce $g(x)=J(x), x \in M_{1 s}$ if $g$ is homogeneous.

Suppose that (16.1) holds. Set $F=M_{1 s}$ and define $\Phi_{1}, \Psi_{1}: M_{1 s} \rightarrow U_{1}$ by $\Phi_{1}(x)=\Psi_{1}(x)=\exp (i x)$, $x \in M_{1 s}$. Also define $\Phi_{2}, \Psi_{2}: M_{1 s} \rightarrow U_{2}$ by $\Phi_{2}(x)=\exp (i f(x))$ and $\Psi_{2}(x)=\exp (i g(x)), x \in M_{1 s}$. As $\exp i M_{j s}=U_{j}$ for $j=1,2$, the maps $\Phi_{j}$ and $\Psi_{j}$ are surjective for $j=1,2$. Apparently, we have

$$
\sigma\left(\Phi_{1}(x) \Psi_{1}(y)\right)=\sigma\left(\Phi_{2}(x) \Psi_{2}(y)\right), \quad x, y \in F .
$$

Then by Theorem 15 there exists a Jordan *-isomorphism $J: M_{1} \rightarrow M_{2}$ and $u_{0} \in U_{2}$ such that

$$
\exp (i f(x))=\Phi_{2}(x)=u_{0} J\left(\Phi_{1}(x)\right)=u_{0} J(\exp (i x))=u_{0} \exp (i J(x))
$$

and

$$
\exp (i g(x))=\Psi_{2}(x)=J\left(\Psi_{1}(x)\right) u_{0}^{-1}=J(\exp (i x)) u_{0}^{-1}=(\exp (i J(x))) u_{0}^{-1}
$$

for every $x \in M_{1 s}$, and hence we obtain (16.2).

Now suppose that (16.4) holds. Then by a simple calculation we have that

$$
\exp (i f(x)) \exp (i g(y))
$$

$$
=u_{0}\left(p J(\exp (i x)) J(\exp (i y))+(1-p) J(\exp (i x))^{-1} J(\exp (i y))^{-1}\right) u_{0}^{-1} .
$$

Using a calculation similar to the one we have applied in the proof of the implication (12.5) $\Rightarrow$ (12.3) in Theorem 12 we have that

$$
r(\exp (i x) \exp (i y)-1)=r(\exp (i f(x)) \exp (i g(x))-1), \quad x, y \in M_{1 s}
$$


and hence we obtain (16.3). In particular, if $f$ is homogeneous, then $f(0)=0$. Thus we have

$$
1=\exp (i f(0))=u_{0}\left(p J\left(\exp (i 0)+(1-p) J(\exp (i 0))^{-1}\right)=u_{0} .\right.
$$

It follows that

$$
\begin{aligned}
\exp (i t f(x))=\exp (i f(t x))=p \exp (i J(t x))+(1 & -p)(\exp (i J(t x)))^{-1} \\
& =p \exp (i t J(x))+(1-p) \exp (-i t J(x)), \quad x \in M_{1 s} .
\end{aligned}
$$

Letting $t \rightarrow 0$, from

$$
(\exp (i t f(x))-1) / i t=p(\exp (i t J(x))-1) / i t+(1-p)(\exp (-i t J(x))-1) / i t,
$$

we deduce

$$
f(x)=(2 p-1) J(x), \quad x \in M_{1 s} .
$$

In a similar manner we obtain $g(x)=(2 p-1) J(x), x \in M_{1 s}$.

Suppose that (16.3) holds. Set $F=M_{1 s}$ and once again define $\Phi_{1}, \Psi_{1}: M_{1 S} \rightarrow U_{1}$ by $\Phi_{1}(x)=$ $\Psi_{1}(x)=\exp (i x)$ and $\Phi_{2}, \Psi_{2}: M_{1 S} \rightarrow U_{2}$ by $\Phi_{2}(x)=\exp (i f(x)), \Psi_{2}(x)=\exp (i g(x)), x \in M_{1 s}$. Then $\Phi_{j}$ and $\Psi_{j}$ are both surjective maps for $j=1,2$. Apparently, we have

$$
r\left(\Phi_{1}(x) \Psi_{1}(y)-1\right)=r\left(\Phi_{2}(x) \Psi_{2}(y)-1\right), \quad x, y \in M_{1 s} .
$$

By Theorem 15 there exists a Jordan *-isomorphism $J$ from $M_{1}$ onto $M_{2}$, a central projection $p \in M_{2}$ and a unitary $u_{0} \in U_{2}$ such that

$$
\Phi_{2}(x)=u_{0}\left(p J\left(\Phi_{1}(x)\right)+(1-p) J\left(\Phi_{1}(x)\right)^{-1}\right)
$$

and

$$
\Psi_{2}(x)=\left(p J\left(\Psi_{1}(x)\right)+(1-p) J\left(\Psi_{1}(x)\right)^{-1}\right) u_{0}^{-1}
$$

hold for every $x \in M_{1 s}$. Then

$$
\exp (i f(x))=u_{0}\left(J(\exp (i x))+(1-p) J(\exp (i x))^{-1}\right)
$$

$$
=u_{0}\left(p \exp (i J(x))+(1-p)(\exp (i J(x)))^{-1}\right)
$$

and

$$
\begin{aligned}
\exp (i g(x))=\left(p J(\exp (i x))+(1-p) J(\exp (i x))^{-1}\right) u_{0}^{-1} & \\
& =\left(p \exp (i J(x))+(1-p)(\exp (i J(x)))^{-1}\right) u_{0}^{-1}
\end{aligned}
$$

for every $x \in M_{1 s}$. This completes the proof.

The following statement is an easy consequence of Corollary 16, one just needs to take $g=f$ (and have a short look at the argument in the proof of Corollary 14 concerning centrality).

Corollary 17. Let $M_{j}$ be a von Neumann algebra for $j=1$, 2. Suppose that $f$ is a bijection from $M_{1 s}$ onto $M_{2 s}$. Then the following two conditions are equivalent:

(17.1) $\sigma(\exp (i x) \exp (i y))=\sigma(\exp (i f(x)) \exp (i f(y))), \quad x, y \in M_{1 s}$;

(17.2) there exists a Jordan ${ }^{*}$-isomorphism J from $M_{1}$ onto $M_{2}$ and a central symmetry $u_{0} \in U_{2}$ such that

$$
\exp (i f(x))=u_{0} \exp (i J(x)), \quad x \in M_{1 s} .
$$

In particular, if $f$ is homogeneous, then we have $f=J$ and $u_{0}=1$.

The following two conditions are also equivalent:

(17.3) $r(\exp (i x) \exp (i y)-1)=r(\exp (i f(x)) \exp (i f(y))-1), \quad x, y \in M_{1 s}$;

(17.4) there exists a Jordan ${ }^{*}$-isomorphism $J: M_{1} \rightarrow M_{2}$, a central projection $p \in M_{2}$ and a central symmetry $u_{0} \in U_{2}$ such that

$$
\exp (i f(x))=u_{0}\left(p \exp (i J(x))+(1-p)(\exp (i J(x)))^{-1}\right), \quad x \in M_{1 s} .
$$

In particular, if $f$ is homogeneous, then we have $f=(2 p-1) J$ and $u_{0}=1$. 
Our last statement is again a simple consequence of Corollary 16, one needs to consider the $\operatorname{map} g(y)=-f(-y), y \in M_{1 s}$.

Corollary 18. Let $M_{j}$ be a von Neumann algebra for $j=1,2$. Suppose that $f$ is a bijection from $M_{1 s}$ onto $M_{2 s}$. Then the following two conditions are equivalent:

(18.1) $\sigma\left(\exp (i x)(\exp (i y))^{-1}\right)=\sigma\left(\exp (i f(x))(\exp (i f(y)))^{-1}\right), \quad x, y \in M_{1 s}$;

(18.2) there exists a Jordan *-isomorphism J from $M_{1}$ onto $M_{2}$ and $u_{0} \in U_{2}$ such that

$$
\exp (i f(x))=u_{0} \exp (i J(x)), \quad x \in M_{1 s} .
$$

In particular, if $f$ is homogeneous, then we have $f=J$ and $u_{0}=1$.

The following two conditions are also equivalent:

(18.3) $r\left(\exp (i x)(\exp (i y))^{-1}-1\right)=r\left(\exp (\right.$ if $\left.(x))(\exp (i f(y)))^{-1}-1\right), \quad x, y \in M_{1 s}$;

(18.4) there exists a Jordan ${ }^{*}$-isomorphism $J: M_{1} \rightarrow M_{2}$, a central projection $p \in M_{2}$ and unitary $u_{0} \in U_{2}$ such that

$$
\exp (i f(x))=u_{0}\left(p \exp (i J(x))+(1-p)(\exp (i J(x)))^{-1}\right), \quad x \in M_{1 s} .
$$

In particular, if $f$ is homogeneous, then we have $f=(2 p-1) J$ and $u_{0}=1$.

\section{REFERENCES}

[1] P. Ara and M. Mathieu, Local Multipliers of $C^{*}$-algebras, Springer-Verlag, London, 2003.

[2] M. Brešar and Š. Špenko Determining elements in Banach algebras through spectral properties, J. Math. Anal. Appl. 393 (2012), 144-150.

[3] O. Hatori, G. Hirasawa, T. Miura and L. Molnár, Isometries and maps compatible with inverted Jordan triple products on groups, Tokyo J. Math., 35 (2012) 385-410.

[4] O. Hatori, S. Lambert, A. Luttman, T. Miura, T. Tonev and R. Yates, Spectral preservers in commutative Banach algebras, in Function Spaces in Modern Analysis, K. Jarosz, Edt. Contemporary Mathematics vol. 547, American Mathematical Society, 2011, pp. 103-124.

[5] O. Hatori and L. Molnár, Isometries of the unitary groups and Thompson isometries of the spaces of invertible positive elements in $C^{*}$-algebras, J. Math. Anal. Appl. 409 (2014), 158-167

[6] R. V. Kadison, A generalized Schwarz inequality and algebraic invariants for operator algebras, Ann. Math. 56 (1952), 494-503.

[7] L. Molnár, Some characterizations of the automorphisms of $B(H)$ and $C(X)$, Proc. Amer. Math. Soc. 130 (2002), 111-120.

[8] L. Molnár, General Mazur-Ulam type theorems and some applications, to appear in a volume of the series "Operator Theory: Advances and Applications".

[9] L. Molnár and P. Szokol, Transformations on positive definite matrices preserving generalized distance measures, Linear Algebra Appl. 466 (2015), 141-159.

[10] T.W. Palmer, Banach Algebras and The General Theory of *-Algebras, Vol. I., Encyclopedia Math. Appl. 49, Cambridge University Press, 1994.

[11] N.V. Rao and A.K. Roy, Multiplicatively spectrum-preserving maps on function algebras, Proc. Amer. Math. Soc., 133 (2005), 1135-1142.

[12] A. R. Sourour, Invertibility preserving linear maps on L(X), Trans. Amer. Math. Soc. 348 (1996) 13-30.

Department of Mathematics, Faculty of Science, Nimgata University, Nitgata 950-2181 Japan

E-mail address: hatori@math.sc.niigata-u.ac.jp

Department of Analysis, Bolyai Institute, University of SZEged, H-6720 Szeged, Aradi Vértanúk tere 1., Hungary and MTA-DE “Lendület” Functional Analysis Research Group, Institute of Mathematics, University of Debrecen, H-4010 Debrecen, P.O. BoX 12, Hungary

E-mail address: molnarl@math.u-szeged.hu

URL: http: //www . math . unideb.hu/ molnarl/ 